\title{
Prévision quantitative des précipitations
}

\author{
Y. Veyrier \\ Equipe Contrôle de la Division Prévision de la Météorologie nationale
}

\section{Rappels sur les modèles numériques de prévision objective utilisés à la Météoro- logie nationale}

Depuis plus d'une vingtaine d'années que la prévision numérique a été introduite à la Météorologie nationale, les performances de ses modèles se sont accrues avec la puissance des calculateurs. Aujourd'hui la Météorologie nationale utilise opérationnellement un CRAY II capable de traiter 500 millions d'opérations par seconde, et la qualité des prévisions à 5 jours d'échéance est équivalente à celle que l'on obtenait numériquement à 24 heures d'échéance dans les années 1950.

\subsection{Les systèmes actuels de prévision}

La nécessité de traiter l'atmosphère sur l'hémisphère (voire la sphère) impose des limites quant aux résolutions spatiale et temporelle que l'on peut utiliser pour les calculs. Or on a besoin d'échelles d'autant plus fines que l'on veut représenter précisément les phénomènes atmosphériques. Ainsi pour prévoir correctement les précipitations doit-on simuler les effets du relief avec précision.

Pour être à même de répondre à ces deux impératifs, la Météorologie nationale a mis en place un système gérant deux modèies imbriqués. Le modèle à grande échelle (maille équivalente de l'ordre de $120 \mathrm{~km}$ de côté à nos latitudes) EMERAUDE, mis en service fin 1984, vient de devenir global (il était hémisphérique jusqu'en juin 1988), tandis que le modèle mésoéchelle (maille voisine de $35 \mathrm{~km}$ de côté) PERIDOT, mis en service en 1985, effectue ses calculs sur un domaine limité à la France et à son environnement (celui-ci a été récemment étendu dans le cadre de l'utilisation du CRAY II) (fig. 1).

\subsection{Le traitement des précipitations dans les modèles}

A ce stade, après simplification aux échelles considérées, la formulation mathématique des lois physiques auxquelles obéit l'atmosphère ne suffit pas pour faire intervenir les phénomènes de plus petites échelles.

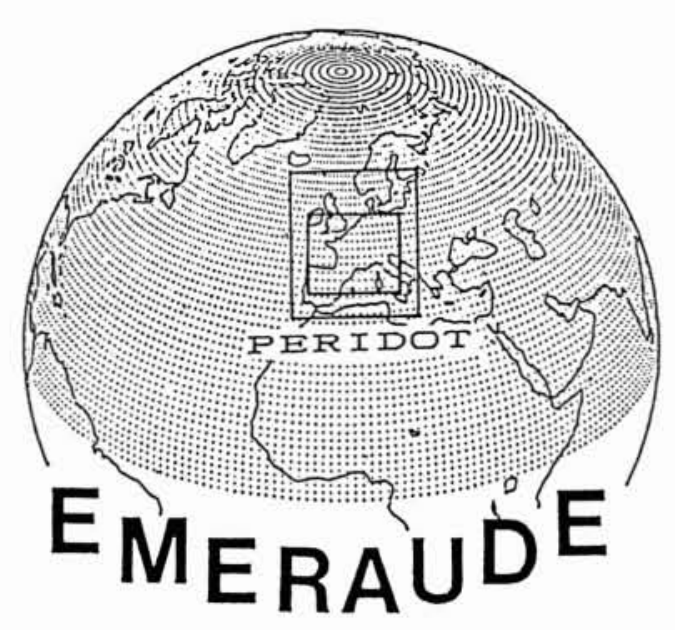

1. Sur cette figure sont représentés les domaines respectifs des modèles du système de prévision de la Météorologie Nationale. Le domaine du modèle EMERAUDE était hémisphérique (il vient de devenir global). Celui du modèle PERIDOT était dans un premier temps limité à la France et son environ. nement proche. Il a été récemment étendu dans le cadre de l'utilisation opérationnelle du calculateur CRAY II.

\section{Quantitative rainfall forecast}

This article is a synthesis about the state of the quantitative rainfall forecast at the State Meteological Observatory. The forecast system is briefly described, whilst especially emphasizing the characteristics of rainfall. Several follow up and quality control results of the numerical amounts of rainfall, mainly derived from the PERIDOT mesoscale model which was put into operation in 1985, are specified. It demonstrates a potential which is opening new perspectives for these types of forecasts. 
Ainsi la condensation et la convection qui jouent un rôle important dans la formation des précipitations doivent être paramétrées à l'aide de schémas assez rudimentaires et souvent empiriques. Les modèles ne gèrent pas explicitement la variable " eau-liquide ", mais génèrent des précipitations dès qu'il $\mathrm{y}$ a sursaturation dans une maille (précipitations de grande échelle). Ils ne décrivent pas non plus explicitement les mouvements verticaux liés aux phénomènes convectifs (orages, lignes de grains,...) mais en simulent les effets d'homogénéisation verticale des grandeurs thermodynamiques et les précipitations convectives éventuelles.

\subsection{Les variables concernant l'humidité prises en compte pour l'initialisation}

La connaissance approfondie et détaillée de l'état initial d'un modèle (schéma d'analyse) conditionne la qualité des prévisions que l'on obtiendra (un processus d'initialisation est nécessaire pour assurer la cohérence de cet état initial avec les hypothèses concernant la physique de l'atmosphère).

Pour analyser l'humidité de l'atmosphère on recense le maximum d'observations disponibles (prédicteurs) qui peuvent lui être reliées statistiquement. Outre les mesures d'humidité (observations au sol et en altitude), on utilise les observations de nuages faites au sol et les données télédétectées issues de satellites (radiances).

\section{L'utilisation opérationnelle et le contrôle des modèles en matière de prévisions quantitatives de précipitations}

\subsection{L'étape essentielle de la démarche du pré- visionniste: interprétation et adaptation des sorties de modèles}

Au fil des progrès réalisés en prévision numérique, l'utilisation des modèles en matière de prévision de précipitations a évolué. Pendant longtemps elle a consisté en l'interprétation des champs d'altitude prévus (géopotentiel, température, humidité,...). En fonction de ses connaissances climatologiques, géographiques et de son expérience, le prévisionniste est en mesure d'en déduire une évaluation de l'intensité des précipitations avec un degré de confiance variable selon l'échéance, le type de temps et le domaine considéré.

Des études d'adaptations statistiques des sorties de modèles ont parallèlement été menées qui, si elles ont donné des résultats intéressants concernant la prévision des températures au niveau du sol, ont été jusqu'alors moins fructueuses pour la prévision des précipitations.

Les premières utilisations des sorties directes de quantités de précipitations ont pris la forme d'évaluations de lames d'eau prévues par bassins dont les résultats, estimés au cours des années 1981 à 1984, se sont montrés moins satisfaisants que ceux obtenus par le prévisionniste (cf. Prévision quantitative de la pluie et assistance météorologique).
Aujourd'hui, pour la courte échéance, le prévisionniste dispose du modèle mésoéchelle PERIDOT comme support complémentaire pour l'interprétation et l'adaptation des prévisions numériques. A partir de l'analyse des prévisions à grande échelle (EMERAUDE) et de sa connaissance précise du temps observé (observations des stations du réseau synoptique, images satellites, images radar), il lui est possible de critiquer la qualité des prévisions à petite échelle (PERIDOT). Il concentre alors son attention sur la description locale de phénomènes précis (précipitations, températures, nébulosités,...), is sue de ce modèle, qu'il peut éventuellement valider. A ce niveau intervient la maîtrise des particularités de ce type de prévisions acquise par l'expérience qu'il en a.

Cette démarche révèle l'importance de la mise en œuvre de contrôles systématiques des divers éléments intervenant, et particulièrement, en ce qui nous concerne, des prévisions quantitatives de précipitations.

\subsection{Contrôle semi-objectif des quantités de précipitations prévues par les modèles EMERAUDE et PERIDOT dans le cadre de leur utilisation opérationnelle}

Au cours des trois premiers mois de l'année 1986 une évaluation systématique des quantités de précipitations prévues par les modèles EMERAUDE et PERIDOT a été menée dans le cadre de leur utilisation opérationnelle. Tous les jours ces prévisions sont disponibles sous la forme de cumuls, en chaque point de grille, portant sur 6 heures et jusqu'à 36 heures d'échéance pour PERIDOT et 96 heures d'échéance pour EMERAUDE. Les isohyètes en résultant sont tracées sur un domaine centré sur la France.

Le problème majeur rencontré tient à la nature complexe du champ de précipitations réelles pour lequel on ne dispose que d'observations quantitatives discrètes. Toutefois l'avantage d'un contrôle en temps réel est que ces données peuvent être valablement critiquées et interpolées avec une relative confiance en les confrontant à d'autres sources de mesures (images radar, images satellites, réseau nivométéorologique en hiver pour les zones montagneuses). On a pu de la sorte obtenir pour chaque situation un tracé des isohyètes observées qui doit cependant être considéré avec prudence.

Les critères de sélection des situations présentées sont axés sur le comportement du modèle PERIDOT, d'une part en rapport avec la situation météorologique et la qualité de sa prévision (bonne ou mauvaise), et d'autre part en rapport avec l'appréciation de l'apport ou de la détérioration éventuelle d'information sur le modèle EMERAUDE.

Cette étude montre que pour des situations de type hivernal, le modèle PERIDOT s'avère être un outil complétant notablement l'information du modèle EMERAUDE. Notamment, une meilleure prise en compte d'effets orographiques d'échelles assez fines (sur le pourtour méditerranéen en particulier) lui permet de diagnostiquer des noyaux d'intensités pouvant dépasser $20 \mathrm{~mm}$ en 6 heures (quand le modèle à grande échelle n'indique que des précipitations faibles) qui sont comparables aux observations et dont la localisation est conforme à la réalité. Dans certains cas de situations de 
PRESSIOK REDUITE AU NIVEAU DE LA KER CAPA FRONTS DE SURFACE
le $23 / 0 \mathrm{~L} / 1980$ A OETU

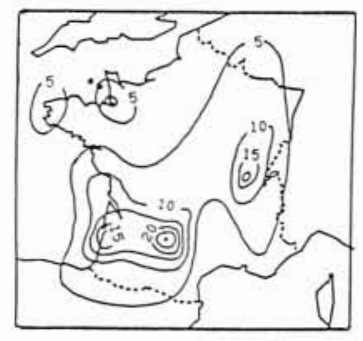

PREVISIOK PERIDOT (MK)

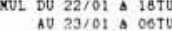

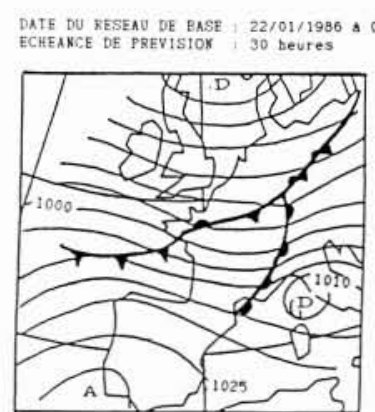

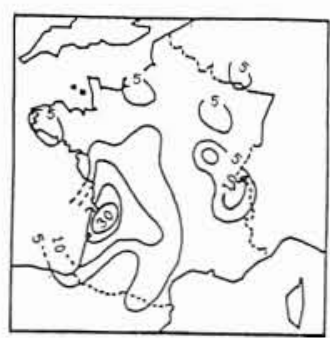

PRECIPITATIOKS OBSERVEES

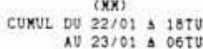

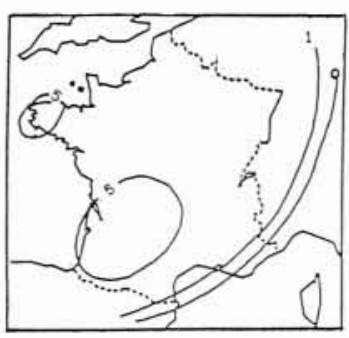

PREVISIOK EKERAUDE (NO CUKUL DU $22 / 01$ \& $18 T$ TV
AV $23 / 01$ \& O6TV
2. Cette situation est caractérisée par le passage d'une perturba tion sur la France soumise à un flux d'ouest rapide.

Le modèle PERIDOT donne une information quantitative complémentaire sur le modèle EMERAUDE. Mais il aurait été intéressant de disposer d'une analyse plus fine des précipitations tombées sur l'ouest du Massif Central pour évaluer l'effet au vent du relief qui semble important.
DATE DU RESEAU DE BASE : $28 / 01 / 1986$ A DOTU ECHEARCE DE PREVISIOS: 30 heures
PRESSIOS REDUITE AU MIVEAU DE LA MER (hPA FRONTS DE SUPFACE

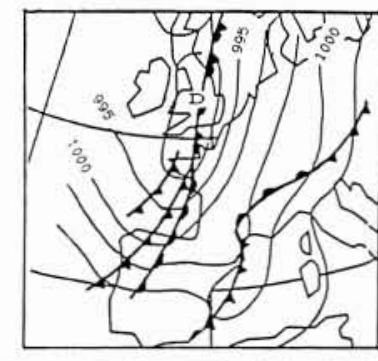

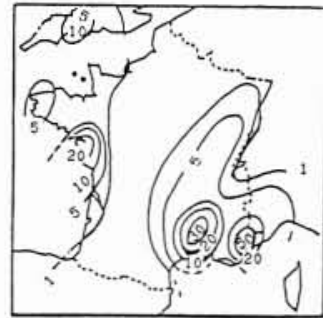

PRECIPITATIONS OBSERVEES

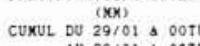

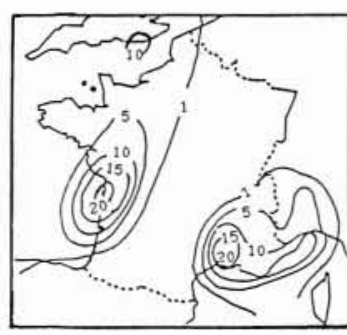

PREVISION PERIDOT (KM)
CXNUL DO $29 / 01$ \& OOTU
AU $29 / 01$ \& OOTU

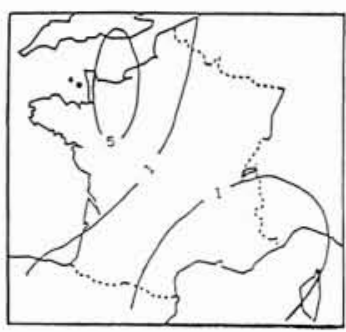

PREVISION EMERAUDE IKO AU 29/01 \& OBT
3. Cette situation météorologique est d'un type classique généralement associé à des précipitations importantes sur le relief des régions méridionales.

L'apport du modèle PERIDOT, lié à sa résolution plus fine sur le modèle EMERAUDE, est très substantiel. En effet ce modèle donne une information appréciable quant à l'exis tence et aux ordres de grandeurs de noyaux plus intenses, même si leur localisation n'est pas parfaite. précipitations à caractère essentiellement frontal, $\mathrm{PE}$ RIDOT semble capable de reproduire des ondulations qui passent au travers de la maille du modèle EMERAUDE.

Cependant l'effet de foehn, se traduisant par des précipitations moindres sous le vent du relief, serait un peu surestimé, tandis que les précipitations au vent du relief semblent parfois localisées un peu trop à l'avant de celui-ci.

Les figures 2 et 3 sont données à titre d'exemples de cette approche et ne sont pas représentatives de toutes les caractéristiques mises en évidence.

\subsection{Contrôles objectifs}

Parallèlement aux contrôles effectués dans le cadre de l'utilisation des modèles, les équipes chargées de leur développement procèdent à des évaluations objectives. L'accent est alors mis sur l'aspect diagnostic dans le but de déceler la nature d'éventuels défauts systématiques auxquels on pourra ensuite remédier.

\subsubsection{Evaluation de la prévision par PERIDOT des paramètres du temps sensible}

L'équipe du CRMD de la Météorologie nationale spécialisée dans ce domaine a édité une synthèse des contrôles systématiques des prévisions par PERIDOT des paramètres du temps sensible près du sol (températures minimales et maximales quotidiennes, précipitations,...) réalisés au cours de l'année 1986.

La nécessité de disposer d'observations avec une densité comparable à celle de la grille du modèle a conduit à avoir recours au réseau synoptique des stations de surface. Celles-ci sont au nombre de 138 réparties sur le territoire français. La méthode a consisté à comparer les prévisions obtenues au point de grille le plus proche de chaque station aux valeurs observées. On rencontre alors deux principaux problèmes. D'une part " le plus proche " traduit parfois un éloignement notable qui peut en outre s'accompagner d'une différence d'altitude non négligeable entre le relief réel et celui du modèle. D'autre part, une étude complémentaire a 


\begin{tabular}{|l|c|c|}
\hline Echeance de prévision & $0 \mathrm{~h}-6 \mathrm{~h}$ & $6 \mathrm{~h}-12 \mathrm{~h}$ \\
\hline Nombre d'observations & 27300 & 39700 \\
Koyenne observée & 0,6 & 0,9 \\
Xoyenne prévue & 0,2 & 0,4 \\
Erreur moyenne (prévision-observation) & $-0,41$ & $-0,48$ \\
Erreur absolue moyenne & 0,62 & 0,91 \\
\hline
\end{tabular}

\begin{tabular}{|l|c|c|}
\hline Echeance de prévision & $24 \mathrm{~h}-30 \mathrm{~h}$ & $30 \mathrm{~h}-36 \mathrm{~h}$ \\
\hline Nombre d'observations & 26800 & 39200 \\
Moyenne observee & 0,7 & 0,9 \\
Moyenne prevue & 0,4 & 0,5 \\
Erreur moyenne (prevision-observation) & $-0,26$ & $-0,45$ \\
Erreur absolve moyenne & 0,71 & 1.07 \\
\hline
\end{tabular}

Les moyennes et les erreurs sont exprimées en mm.

4. Tableau de statistiques annuelles (1986) de cumuls en 6 heures de précipitations observées et prévues par le modèle PERIDOT aux stations du réseau synoptique français d'ob. servations météorologiques (tiré de Evaluation du modèle à maille fine Péridot).
EVENEMENTS : $\mathrm{A}=$ PLUIE, $\mathrm{NA}=$ NON PLUIE

\begin{tabular}{|c|c|c|c|c|}
\hline PIO & & A & NA & \\
\hline A & 12 & 122 & 8329 & $20 \quad 451$ \\
\hline \multirow[t]{2}{*}{ NA } & 2 & 373 & 11121 & 13494 \\
\hline & 14 & 495 & 19450 & 33945 \\
\hline
\end{tabular}

pourcentage de previsions correctes $=68 \%$ pourcentage de detections $=84 \%$ pourcentage de fausses alertes $\quad=41 \%$ indice de qualite (ROUSSEAU) $=0,37$

EVENEMENTS : CLASSES DE HAUTEURS EN MM

\begin{tabular}{|r|r|r|r|r|r|}
\hline P $\backslash 0$ & 0 & $0-1$ & $1-5$ & $5-10$ & $>10$ \\
\hline 0 & 16350 & 1837 & 364 & 96 & 76 \\
$0-1$ & 7622 & 3230 & 923 & 181 & 108 \\
$1-5$ & 603 & 983 & 681 & 159 & 98 \\
$5-10$ & 72 & 168 & 115 & 61 & 31 \\
$>10$ & 32 & 63 & 48 & 24 & 20 \\
\hline
\end{tabular}

nombre d'observations $\quad=39174$ indice de qualite (ROUSSEAU) $=0,51$

5. Tableaux de contingence des variables "pluie observée", "pluie prévue ", la pluie étant cumulée sur 6 heures et la prévision étant celle du modèle PERIDOT pour l'échéance 30 heures - 36 heures (tiré de Evaluation du modèle à maille fine Péridot). montré qu'une part des erreurs constatées s'explique par la variabilité spatiale des paramètres météorologiques qui, à l'échelle inférieure à la maille du modèle, est en quelque sorte un seuil de prévisibilité (cf. Evaluation $d u$ modèle à maille fine Péridot). En effet, une valeur prévue au point de grille est représentative d'une moyenne sur un domaine de dimension égale à la maille du modèle. Elle n'est donc pas directement comparable à la valeur mesurée à la station.

On doit aussi tenir compte des erreurs de mesures et de transmissions qui n'ont été que partiellement filtrées.

Les statistiques concernant les précipitations sont particulièrement sensibles à ces problèmes et doivent être interprétées avec prudence.

Les erreurs moyennes montrent un déficit systématique des précipitations du modèle, accentué dans les premières échéances (problème du "spin up "), et moindre la nuit (sans doute parce que les précipitations convectives sont dans la réalité aussi plus faibles la nuit) (fig. 4).

Les écart-types des erreurs sont difficilement utilisables du fait de la méconnaissance de l'écart-type des précipitations dans la maille (qui n'a d'ailleurs peut-être pas de sens).

L'indice de Rousseau calculé à partir des tableaux de contingence des prévisions par seuils montre une qualité nettement meilleure qu'une prévision de type climatologique. L'analyse plus détaillée fait apparaître une discrimination moyenne des événements " pluie " et " non pluie » (68\% de prévisions correctes), qui s'accompagne d'un taux de fausses alertes de l'ordre de $41 \%$ (ces résultats sont ceux obtenus pour le cumul entre les échéances 30 heures et 36 heures). Elle révèle aussi des prévisions trop fréquentes de petites pluies $(\leqslant 1 \mathrm{~mm})$ alors qu'il ne pleut pas, et le biais négatif obtenu sur les quantités semble dû à une nette sous-estimation des fortes pluies (fig. 5).

\subsubsection{Evaluation d'une simulation du modèle PE- RIDOT sur les USA}

La synthèse de l'évaluation semi-objective évoquée n'a porté que sur les trois premiers mois de l'année 1986 car au cours du printemps, et surtout de l'été, on s'est aperçu d'une dégradation sensible de la qualité des prévisions de précipitations reliée à des défaillances encore importantes du schéma de paramétrisation de la convection. Il n'est donc pas surprenant que les contrôles objectifs qui avaient porté sur toute l'année donnent une vision plus pessimiste de la qualité des prévisions.

On touche là à un problème important en matière de prévision numérique des précipitations à mésoéchelle qu'a mis en évidence l'évaluation d'une simulation du modèle PERIDOT sur les USA. En effet, cette étude du comportement du modèle sur une situation (22 avril 1981) pour différentes résolutions (mailles de 160, 80, 40 $\mathrm{km}$ ) et deux schémas classiques de convection (KuoGELEYN, BougEaULT) souligne l'importance d'une paramétrisation soignée de la convection profonde à ces échelles. 


\section{Conclusion et perspectives}

Les résultats présentés des contrôles des prévisions numériques de précipitations permettent d'apprécier les progrès réalisés et les perspectives pour l'avenir, tout en fixant les limites de l'utilisation de ce type de produits.

En effet, le modèle mésoéchelle PERIDOT est à même désormais de diagnostiquer les prévisions de paramètres du temps sensible au niveau du sol. Mais on rencontre alors le problème du mode d'exploitation de ces données. Ainsi, les comparaisons directes des quantités prévues aux quantités mesurées confirment la nécessité d'intégrer ces nouveaux produits dans la démarche suivie par le prévisionniste d'interprétation et de validation des sorties de modèles. Une méthode de contrôle conforme à cette démarche a mis en évidence les qualités comme outil complémentaire du modèle PERIDOT. Des évaluations plus fines du comportement des modèles révèlent aussi les défauts systématiques que l'on doit corriger.

La méthodologie des contrôles qui seront développés devra donc viser à satisfaire trois objectifs.

Il faut bien sûr assurer le suivi de l'évolution de la qualité des prévisions que l'on fournit. Pour ce faire il est nécessaire de coder l'information de telle sorte que l'on dispose de données comparables. Les problèmes de qualité des mesures peuvent être en partie résolus par l'utilisation de fichiers critiqués (fichiers climatologiques pour des contrôles à long terme, ou fichiers opérationnels mais moins fournis utilisés pour le suivi au jour le jour des bilans hydrologiques). Les difficultés relatives à la représentativité des divers types de données peuvent être surmontées en considérant des domaines géographiques appropriés (bassins versants, maille du modèle) et différentes périodes de cumuls.

Pour tirer le meilleur profit du modèle, il est impératif d'en connaitre au mieux le comportement et les particularités. Un suivi systématique dans le cadre de l'utilisation opérationnelle apporte des éléments de réponse que l'on ne sait actuellement pas évaluer autrement. Dans cette même optique, on peut envisager un contrôle orienté vers la recherche des utilisations les plus adaptées aux prévisions que l'on peut fournir. Par exemple, plutôt que de fixer $a$ priori le point de grille que l'on assimilera à une station d'observation, on peut chercher à déterminer le plus représentatif de la station en question pour la prévision des précipitations. On notera qu'une étude d'adaptation statistique des sorties du modèle PERIDOT est en cours.

Enfin du fait même du caractère empirique des schémas de paramétrisation des phénomènes physiques intervenants, il est indispensable pour les améliorer d'en mesurer le plus précisément possible les qualités comme les défauts. Ces derniers ne pourront être corrigés qu'une fois que l'on aura pu les isoler. Pour cela il est nécessaire d'associer à la batterie des diagnostics existants ceux concernant la prévision des précipitations qui répondent à cet objectif. Ainsi pour analyser correctement le processus du schéma de convection en action, il faut pouvoir séparer les domaines et les situations météorologiques où il intervient de manière prépondérante.

\section{Bibliographie}

Le modèle de prévision numérique Péridot - Formulation dynamique et modes de fonctionnement, M. IMBARD, A. JoLY, R. JUVANON du VACHAT. Note de travail de l'Etablissement d'études et de recherches météorologiques (EERM) $n^{\circ} 161$ (juillet 1986).

Evaluation du modèle à maille fine Péridot - Comparaison avec le modèle Emeraude, J.M. AudoIN, D. RousSEAU, R. JUVANON du VACHAT. Note de travail de l'EERM n ${ }^{\circ} 184$ (mai 1987).
Evaluation d'une simulation du modèle Péridot sur les U.S.A., P. Degardin, M. Imbard, Note de travail de l'EERM n ${ }^{\circ} 192$ (septembre 1987).

L'analyse objective Péridot, Y. Durand, P. Bougeault, Note de travail de l'EERM n ${ }^{\circ} 193$ (octobre 1987).

Prévision quantitative de la pluie et assistance météorologique, A. LE Trocquer. Guide de prévision des crues, tome 2, groupe de prévision des crues, tome 2, Groupe de prévisions des crues (S.H.F.), (1987).

Adresse de l'auteur

Monsieur Y. Veyrier

Equipe Contrôle, Division Prévision

Météorologie nationale

2, avenue Rapp

75340 Paris Cedex 07

Tél. : 45.55.95.02 
M. DUBAND : 3 questions : est-ce que la Météorologie Nationale a abandonné délibérément le recours à des modèles statistiques, en dehors de l'adaptation à des sorties de modèles ? D'autre part, quelle est la référence spatiale des contrôles que vous utilisez? Enfin, quel est le nombre de pluviographes dont dispose la Météorologie nationale?

M. VEYRIER : Avant le développement de Péridot, nous avions les mêmes diagnostics mais sur des modèles à grande échelle qui donnaient des résultats difficiles à utiliser. Les méthodes statistiques sont développées, mais l'investissement principal se fait sur les modèles numériques, coûteux en temps de calcul. Pour des raisons opérationnelles, le nombre de pluviographes utilisés pour les contrôles est d'une centaine : celui du réseau synoptique des stations de surface. Mais nous allons aussi développer des contrôles a posteriori avec le réseau secondaire.

M. DUBAND: Vous parlez de contrôles mensuels. Ce qui me choque est que vous ne fassiez pas référence à la climatologie journalière (de 6 à 24 h.).

M. VEYRIER: Le problème se trouve, d'une part, dans l'estimation du champ réel de précipitations et, d'autre part, dans le fait que nous ne disposons pas d'archives numériques des sorties du modèle.

M. THIRRIOT: A propos de la prise en compte approchée de l'hétérogénéité de la maille, est-il possible d'envisager l'utilisation des méthodes de prise de moyenne à l'échelle de la maille en introduisant des hypothèses de fermeture, comme cela est fait en turbulence ou dans la théorie des mixtures ou mélange?

M. VEYRIER: Je ne suis pas spécialiste de la question. Je voudrais dire que Péridot ne peut décrire les phénomènes généraux de l'atmosphère à une échelle de l'ordre de $50 \mathrm{~km}$, mais il peut à cette échelle décrire des précipitations liées à l'orographie par exemple. Actuellement, au niveau de la recherche, des modèles à mailles plus fines sont testés.

M. OBLED: Au niveau du contrôle, vous avez présenté plusieurs cartes comparant des pluies en $6 \mathrm{~h}$ observées et de pluies calculées "Péridot ". Or le modèle Péridot a des mailles de l'ordre de $1000 \mathrm{~km}^{2}$. Pouvez-vous préciser $s i$, pour ces comparaisons, vous avez étendu les valeurs aux stations pour les rendre moyennes sur $1000 \mathrm{~km}^{2}$ ou vous avez transformé les valeurs Péridot en valeurs ponctuelles?

M. VEYRIER : Les contrôles se font par images. Nous obtenons pour les précipitations réelles et les prévisions par maille un tracé d'isolignes manuel qui est valable à l'échelle régionale. Comme il n'est pas possible en fonctionnement opérationnel de représenter la situation réelle finement, ce sont ces cartes qui sont fournies au prévisionniste, et qui ont été utilisées pour apprécier le modèle Péridot. 